\title{
Nonorganic Vision Loss
}

\author{
Ashwini Kini, ${ }^{1}$ Mangayarkarasi Thandampallayam Ajjeya ${ }^{1}$ and Padmaja Sudhakar ${ }^{1,2}$ \\ 1. Department of Neurology, University of Kentucky, Lexington, KY, USA; 2. Department of Ophthalmology, University of Kentucky, Lexington, KY, USA
}

DOI: https://doi.org/10.17925/EOR.2020.14.1.26

$\mathrm{E}$ very neurologist, ophthalmologist or neuro-ophthalmologist will encounter patients with nonorganic vision loss at some point in their practice. The challenging issue is how to demonstrate normal visual function with objective evidence, while making sure not to miss organic causes of vision loss. The evaluation of such patients could be time consuming and frustrating, both for the patient and the examiner. There are multiple tests that help to concur normal visual functions in such patients. It is important determine baseline visual acuity, and to evaluate the pupils, anterior segment and the fundus, while estimating degree of visual field loss or the absence of field loss. supplementary tests, like automated perimetry, optical coherence tomography, electroretinogram and visual evoked potentials, may be used in the assessment of these patients. Once the diagnosis is established, revealing the diagnosis to the patient and the family can be tricky and can often cause mistrust and frustration, and can pave pathways for medicolegal issues if not handled adeptly. Often, unknown to the physician, there may be hidden psychological conflicts and history of abuse that could be an underlying factor. Hence, no matter how frustrating it is to the physician, a gentle approach is always beneficial. In this article, we will discuss a few clinical and diagnostic methods of evaluating patients with nonorganic vision loss.

\section{Keywords}

Nonorganic vision loss, functional vision loss, visual field loss, distance doubling test, fogging, vertical prisms

Disclosures: Ashwini Kini, Mangayarkarasi Thandampallayam Ajjeya and Padmaja Sudhakar have no financial or non-financial relationships or activities to declare in relation to this article.

Review Process: Double-blind peer review.

Compliance with Ethics: This study involves a review of the literature and did not involve any studies with human or animal subjects performed by any of the authors. The individuals featured in the photographs are members of the authors department and have given full permission for the publication of their images. Consent from the patient discussed in the index case was obtained.

Authorship: The named authors meet the International Committee of Medical Journal Editors (ICMJE) criteria for authorship of this manuscript, take responsibility for the integrity of the work as a whole, and have given final approval for the version to be published.

Access: This article is freely accessible at

touchOPHTHALMOLOGY.com

(C) Touch Medical Media 2020.

Received: 11 June 2020

Accepted: 14 September 2020

Published Online: 9 November 2020

Citation: European Ophthalmic Review. 2020;14(1):26-33

Corresponding Author: Padmaja Sudhakar,

740 S Limestone, Lexington, KY 40536, USA.

E:psu224@uky.edu

Support: No funding was received in

the publication of this article.
Nonorganic vision loss (NOVL) or functional vision loss (FVL) is the presence of abnormal vision, or visual fields, without an organic pathology, despite a thorough and comprehensive evaluation. The symptoms will be inconsistent with the results of an objective examination. ${ }^{1-3}$ In some cases, vision loss is intentionally feigned, while in others it occurs under unconscious conditions.

The diagnosis of NOVL/FVL requires the establishment of findings on clinical examination that prove the integrity and functioning of the visual system. Yet this could be difficult to establish, and potentially time consuming. It is more challenging to diagnose NOVL/FVL when superimposed on organic pathology, but using anatomical rules and basic ophthalmology tools that are not intuitively understood by the patient, one can demonstrate integrity of the visual system and confirm the diagnosis of NOVL or FVL. It is important to remember that many things can present without an organic basis in ophthalmology, such as nystagmus, gaze palsy or ptosis, but in this article, we will restrict our discussion to cases of visual acuity and visual field loss. We will discuss the tests that are most commonly used in clinic to establish the diagnosis.

\section{Index case}

A 17-year-old boy was brought in to the neuro-ophthalmology clinic complaining of subacute vision loss in his right eye that has been painless and progressive for the past several months. During this period, he had seen several physicians, including his primary carer, a general ophthalmologist, and retina and glaucoma specialists, and was ultimately told he had nothing wrong in his eyes. The patient's mother was very frustrated and angry that a psychiatry referral was requested. consequently, primary care requested a neuro-ophthalmology consultation.

During an interview, with his mother present, the patient demonstrated reduced speech and avoided answering questions on underlying stressors. When interviewed alone, he did disclose some domestic disharmony, but refused to answer further questions. On examination, visual acuity in the right eye was 20/100, and the left eye was 20/20. Pupil examination revealed no afferent pupillary defect. When colour vision was checked, the patient read all plates with the left eye, and no plates, including the test plate, with the right eye. The physician also noted that when he sat to the right of the patient and showed him the plates from right side, the patient did not attempt to turn his head to look at it, but was able to read from his steady position. Anterior and posterior segment examination were normal.

Visual field by confrontation revealed a 'tunnel field' that was constricted at a distance of 1 foot from the patient, and stayed at a similar constriction when repeated from 4 feet away. A Humphrey visual field showed small constricted clover leaf field in right eye and normal field in left eye. This was repeated with both eyes open and continued to reveal a constricted clover leaf field in the right eye. An electroretinogram (ERG) and several magnetic resonance images (MRIS) of the brain 
Table 1: Relationship of stereopsis to visual acuity ${ }^{14}$

\begin{tabular}{|l|l|l|}
\hline Stereopsis (arc second) & Visual acuity & Visual acuity \\
\hline 40 & $20 / 20$ & $6 / 6$ \\
\hline 43 & $20 / 25$ & $6 / 7.5$ \\
\hline 52 & $20 / 30$ & $6 / 9$ \\
\hline 61 & $20 / 40$ & $6 / 12$ \\
\hline 78 & $20 / 50$ & $6 / 15$ \\
\hline 94 & $20 / 70$ & $6 / 24$ \\
\hline 124 & $20 / 100$ & $6 / 30$ \\
\hline 160 & $20 / 200$ & $6 / 60$ \\
\hline
\end{tabular}

and orbit had been recently conducted, which were normal and hence were not repeated.

\section{Prevalence of nonorganic vision loss/functional vision loss}

Patients with NOVL are often referred to the neuro-ophthalmology clinic after other providers have done multiple clinical examinations and an extensive work-up that has failed to reveal an underlying cause for poor vision. NOVL could comprise around $1-5 \%$ of the patient population in an ophthalmology clinic, according to some studies, 4,5 and can include both paediatric and adult patients. Mean age at presentation is reported to be around 11-13 years in the paediatric group, and around 40 years in adults, with a fall in incidence after the sixth decade. ${ }^{6,7}$ There is female predominance in both age groups. Around $60-80 \%$ cases are bilateral. Visual acuity combined with visual field loss is found to be the most common presentation in NOVL. ${ }^{6,7}$

\section{Risk factors}

A large proportion of individuals with NOVL could have underlying stressors, psychiatric disorders, attention deficit hyperactivity disorder, somatoform disorders, behavioural/learning disorders or conflicts/ disharmony at home. In children, sexual and physical abuse could be significant risk factors. ${ }^{7.8}$ Likewise, health events (self-limiting illness, operations, injuries) may occur immediately prior to the onset of symptoms. Underlying psychiatric illness is found to be more prevalent in the adult population than the paediatric age group. ${ }^{7}$ On the other hand, patients with NOVL could be without any underlying psychiatric disorders. ${ }^{9-12}$ According to Bruce and Newman, NOVL is not a diagnosis of exclusion, as it is popularly believed, but needs examination and tests to establish normal visual function. ${ }^{11}$ Of note, $15-50 \%$ of the patients with NOVL could have underlying organic visual co-morbidities and fall under 'nonorganic overlay'.? These cases need to be carefully evaluated and managed in order to not miss a subtle, underlying, treatable cause..$^{711}$

\section{Tests to identify nonorganic vision loss in clinic}

The first step is to determine if there is visual acuity loss or visual field loss, and subsequently, whether there is monocular or binocular vision loss. If visual field loss is reported, determine which eye is affected and the area of visual field loss. Complaints of bilateral vision loss could be more challenging to work-up and may require additional testing or follow-up visits before establishing a diagnosis of NOVL/FVL.

\section{Commonly used visual function tests}

Here, we discuss some commonly used tests in practice. These need not be conducted in any specific order. Initial examination starts even as the patient walks into the clinic. Observing them manoeuvre around obstacles could give a fair idea on their level of vision. A patient walking into the clinic with sunglasses on is reported as a strong predictor of NOVL, provided the patient does not have particular indication such as intense photophobia or acute migraine attack..$^{13}$ By offering a spontaneous handshake either at the beginning of the visit or at the end, intentionally in the blind field (in case of field loss), one can observe if patient reaches out without difficulty.

Patients referred for NOVL will have already had their eyes examined multiple times by other providers, and could be frustrated if visual acuity is checked again. This could be very tricky when trying to establish a doctor-patient relationship and could affect any further additional testing or counselling. A few ways to do this differently are listed here. Again, these could have variable results and are not fool-proof.

\section{Snellen's visual acuity and use of fogging}

It is helpful to start checking the vision from the smallest letter available on chart, e.g. 20/15 line, keeping the affected eye open and the good eye closed. This test can be conducted with both eyes open in case of binocular visual loss. Move up the chart by providing encouragement to the patient for every letter read.

Fogging could be tried in patients with monocular vision loss, during which higher strength lenses are placed in front of the good eye with an attempt to blur the vision in the good eye. At the beginning of the test, the patient is informed that their good eye is being checked to improve vision further, and while the test is being performed, consider holding a light conversation with the patient to distract them from being conscious or aware. Ask the patient to read the vision chart after fogging the good eye. The patient unknowingly shifts to reading from their 'bad eye'. This test is usually done in ophthalmology clinics with a phoropter. If a phoropter is not available, a trial frame with a few loose plus lenses may be handy. Additionally, lenses that are the equivalent of plain glass, when combined, can be placed over the patient's glasses or refraction to suggest that these will magnify the letters on the chart. This can be especially useful in binocular visual loss.

\section{Red-green glasses}

This test is based on the principle that coloured lenses allow only similarly coloured light to pass through. The eye with the red filter sees only the images that are red, and similarly, the eye with the green filter sees only images that are green. The patient is given the red green glasses to wear and is presented with red and green letters of gradually increasing size on the Snellen's visual acuity chart. This could be presented to the patient as a colour vision test. Vision in each eye can be checked with reasonable accuracy with this test.

A similar test involves using polarised glasses, with one axis at 90 degrees and the other at 180 degrees in each lens. The patient is asked to read a polarised eye chart with some letters perceptible only to one eye or the other.

\section{Stereopsis and visual acuity correlation}

Stereopsis tests, such as Titmus test or the Randot ${ }^{\circledR}$ test (Stereo optical Co., Inc., Chicago, IL, USA), are based on the principle of binocular fusion and require a particular minimum level of vision to correlate to the degree of stereopsis (Table 1). ${ }^{14}$ These tests can be administered to the patients without raising suspicion, and can be used in patients with monocular and also in binocular loss to some extent." Some studies, however, state that commonly used visual acuity estimates based on stereo acuity might overestimate visual acuity. ${ }^{15}$ 
Figure 1: Eliciting optokinetic nystagmus in binocular (A) and monocular (B) nonorganic vision loss
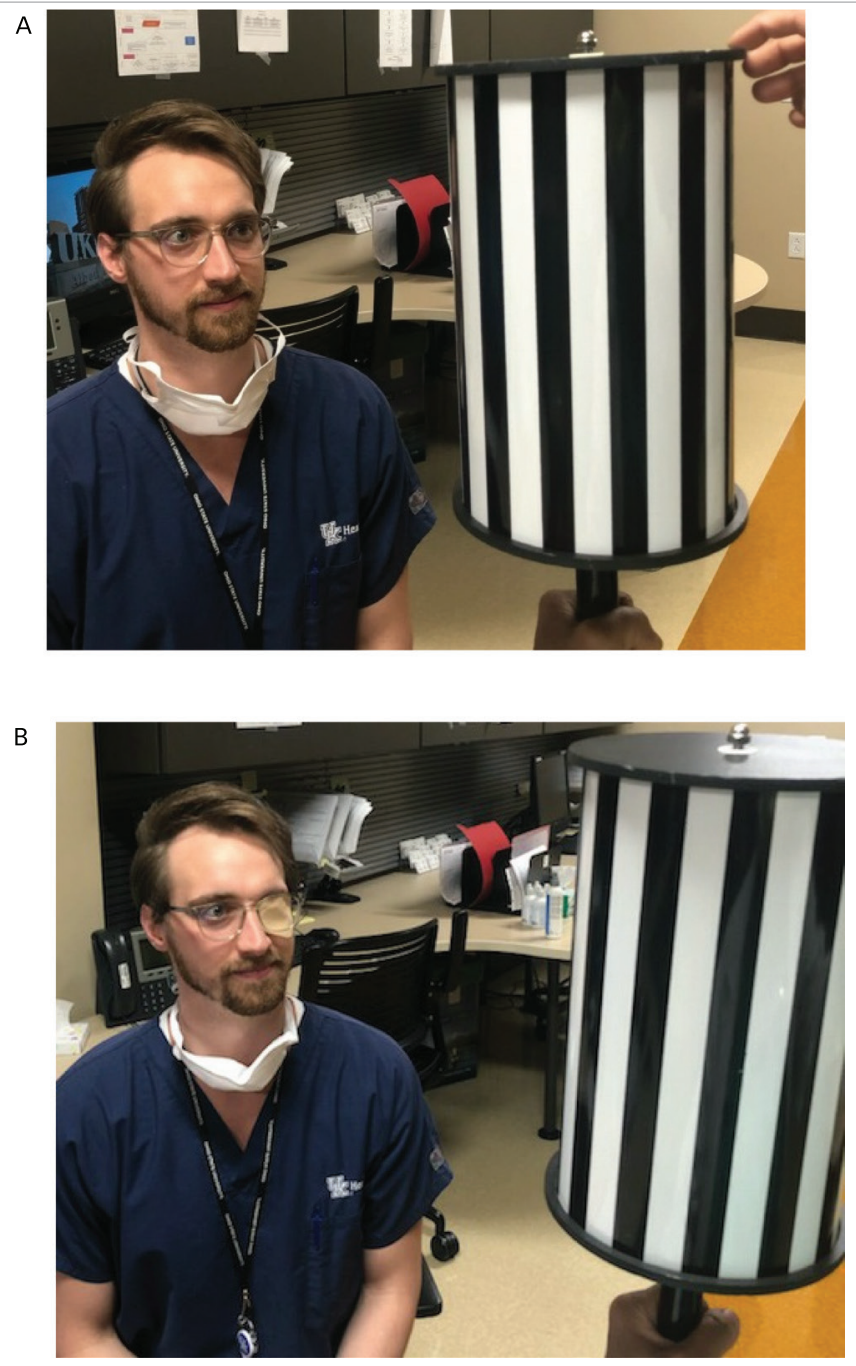

\section{Distance doubling test}

Zinkernagel and Mojon reported high sensitivity and specificity with the distance doubling test. ${ }^{16}$ This test is based on the principle that when vision is checked at a particular distance, for example 20/100 level, when the distance is reduced by half, the patient should be able to read a letter of 20/50 size. Patients with NOVL often claim static, unchanged level of vision despite coming closer to the chart. ${ }^{11,16}$

\section{Using the optokinetic drum}

The optokinetic drum can be used to elicit optokinetic nystagmus in the viewer's eye (there is usually smooth pursuit with refixation saccade). In a patient who claims to be unilaterally or bilaterally blind, to the extent of hand motions or worse, eliciting a nystagmus with the optokinetic drum establishes a vision of at least 20/400. In bilaterally blind patients, the test is conducted with both eyes open; and when unilateral, the test is started with both eyes open and the examiner introduces an occluder in front of the good eye during the course of the test to elicit nystagmus in the claimed blind eye (Figure 1). ${ }^{17}$

\section{Vertical prism test}

This test was first described by Slavin, and involves placing a vertical prism of four prism dioptres, base-down, in front of the good eye..$^{18}$ This is useful in patients with monocular vision loss. Prior to the test, the physician
Figure 2: Patient's perspective of the Snellen's chart when a vertical prism is placed in front of one eye
A E D F C Z P

E D F C Z P

\section{B E D F C Z P}

A. Shows a 20/40 line on Snellen's chart from the perspective of a patient with normal and equal vision. The patient sees both lines with letters that are equally clear; $B$. Shows true organic vision loss in one eye. The better eye sees the clear images and the worse eye sees the blurred images.

should explain to the patient that they will only check the good eye with a prism, and that the prism may split the image. The prism, placed in front of the good eye, makes the image appear higher than it is, and the affected eye sees the original image lower down. The patient is shown 20/40 optotype on a Snellen chart at the standard distance. If the patient describes seeing two images of similar clarity, the patient has unknowingly declared that the vision in his blind eye is at least 20/40, or maybe even better (Figure 2). Golnik et al. used this test in three groups of patients: those with normal vision, organic vision loss, and in those referred with NOVL. They concluded that the vertical prism test was a fairly accurate and reliable test to identify NOVL, and in fact, appropriate image response of one image was detected in some patients referred with NOVL, who, on further work-up, were identified to have other causes of vision loss. ${ }^{19}$

A four-dioptre prism may also be placed base-out in front of the eye with alleged visual reduction, while fixating a 20/40 Snellen letter, eliciting diplopia to detect nonorganic vision loss. When the prism is quickly removed, if movement of the affected eye is detected by the examiner, an acuity, approximately equal to the Snellen letter, is likely.

A slight variation of the vertical prism test can be achieved by blocking the affected eye with a finger or occluder. Then, place the vertical prism base-down splitting the pupil of the good eye (Figure 3A). The patient will report seeing two lines of letters with the good eye from monocular diplopia. Ask the patient to read the bottom line (bottom image) and while they do that slide the prism down over the good eye and remove the occlusion from the affected eye (Figure 3B). The patient will still report seeing two lines of letters, the bottom line is now seen with the affected eye.

\section{Visual surprise}

The menace reflex is reflex blinking that occurs in response to rapid approach of an object. This can be used to detect NOVL; however, this test can be considered controversial, as it can be regarded as an action of threat. Moreover, the perception of motion could be relatively preserved, even in cortically blind patients, which is referred to as the Riddoch phenomenon. ${ }^{20}$ Hence, the menace response cannot be used to rule in or rule out NOVL, as some of these patients could have an underlying visual disorder that could go undetected. ${ }^{21}$

\section{Relative afferent pupillary defect}

In a patient reporting poor vision in one eye, it would be expected to have a relative afferent pupillary defect (RAPD) in that eye if there was an optic neuropathy or a retinal pathology, such as central retinal artery occlusion, accounting for the vision loss. In a patient displaying unilateral profound vision loss, to the extent of no light perception, the absence of RAPD in the presence of normal ophthalmic examination should raise suspicion for NOVL/FVL. This would be difficult to assess when the complaint is bilateral or unilateral with subtle loss, or when there is homonymous hemianopia. Absence of RAPD may need to be verified 
Figure 3: A variation of the prism test by use of an occluder on the affected eye
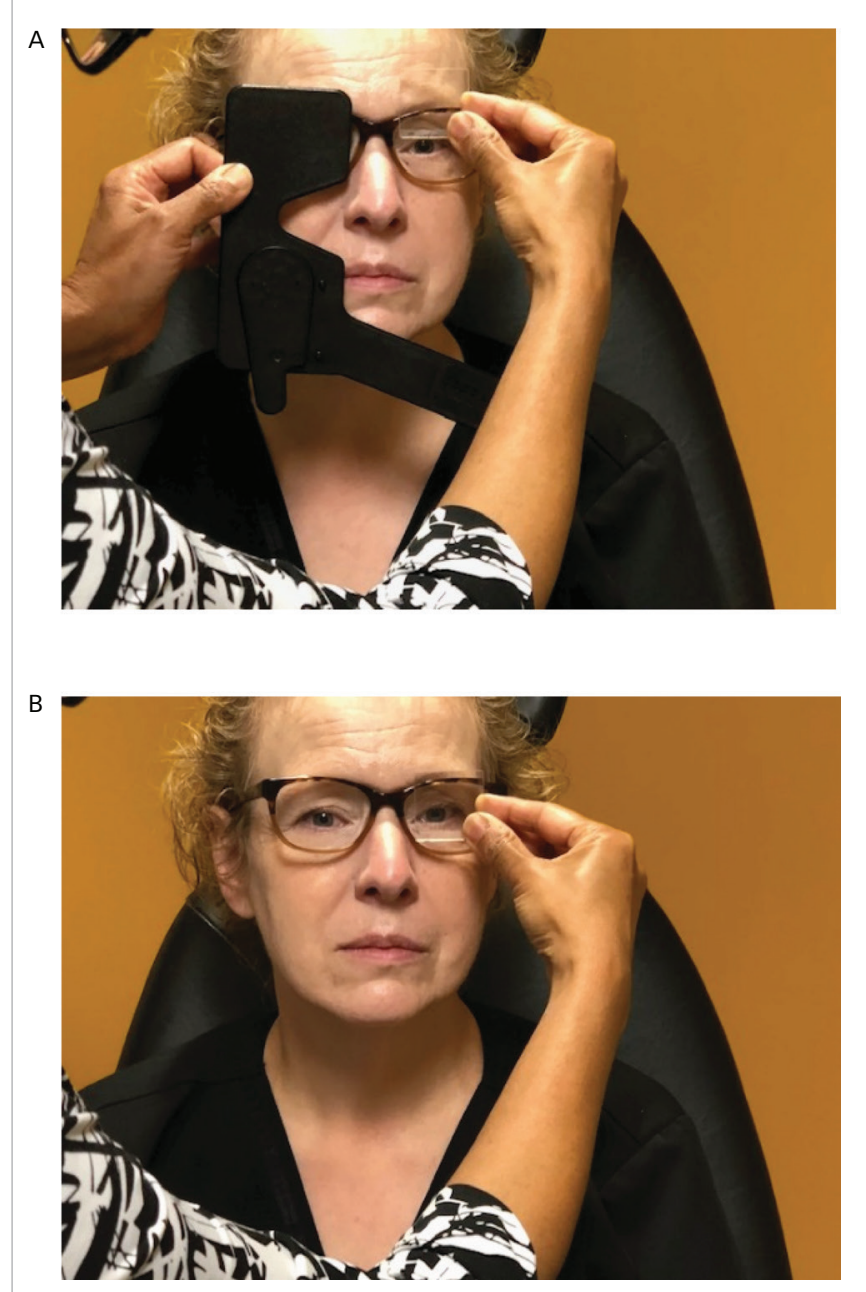

A. Shows occlusion of the affected eye and the placement of the vertical prism, base-down, bisecting the pupil of the unaffected eye, producing monocular vertical diplopia; B. Shows removal of occlusion from the affected eye and movement of the the vertical prism base-down over the unaffected eye, eliciting binocular vertical diplopia.

using neutral density filters, as subtle RAPD can be missed. Similarly, no RAPD is seen in cases of media opacities, like corneal opacity or cataract, in cases of efferent pupillary dysfunction, refractive error, amblyopia or cortical pathology. Lastly, assessment of RAPD may be difficult in the presence of anatomic pupillary abnormality, tonic pupils, absence of an eyeball, pre-existing blindness or profound visual loss in one eye. In some situations, assessment for RAPD by the reverse (looking at pupillary response of the opposite eye), might be helpful.

Foroozan and Lee have discussed that absent RAPD in a structurally normal eye could easily be mislabelled as 'faking' in the presence of true underlying cortical visual field deficit, detected by formal visual field testing.22 This may be seen in chiasmal or retro chiasmal lesions, which would not have any structural eye abnormality, and could display 20/20 vision in both eyes. RAPD may not be apparent, and, therefore, may be missed. Other subtle defects can also be missed when using confrontation technique in the clinic, unless automated perimetry is performed. Sometimes, RAPD and bowtie optic atrophy may be seen on the contralateral side of an optic tract lesion. Optic tract lesions could be missed on clinical examination and on MRI. More specific studies, either with use of contrast on MRI, positron emission tomography (PET), single-photon emission computed tomography (SPECT) or even diffusion tensor imaging or tractography, may be required for further investigation. ${ }^{22-26}$ Clinical presentation and past history could help guide the physician in ordering the right imaging study for the patient.

A useful point to remember is that patients with early Leber's hereditary optic neuropathy may have normal pupil response without RAPD in the background of poor vision and a normal eye examination. ${ }^{27} \mathrm{~A}$ positive family history, central scotoma and bilateral involvement could be important clues to prompt genetic testing in these individuals.

\section{Potential acuity meter}

The potential acuity meter, or interferometer, can evaluate visual acuity prior to cataract surgery so that patients can be furnished with a realistic visual prognosis, pre-operatively. It uses a monochromatic light source that generates interference stripes posterior to the lens, and which are therefore not affected by lenticular opacities. This test can also be used in NOVL. ${ }^{28}$ The patient is told that the test is designed to circumvent the current eye problem and estimate what the vision would have been like had the illness not occurred. It could measure the visual acuity in monocular or binocular visual complaints.

\section{Mirror test}

When distracting the patient by holding a conversation with them, use a mirror that is moved across their field of vision. It is difficult for a patient to resist looking at their own reflection or following the moving image. ${ }^{11}$ This test is useful in patients with proclaimed profound vision loss to light perception or no light perception. It could potentially be used in unilateral or bilateral loss, but one would have to occlude the good eye during the testing.

\section{Test of proprioception}

A patient with bilateral NOVL may be asked to touch the tips of both their fingers keeping their eyes open or with one eye (the good eye) patched, and may be seen to fail this attempt. ${ }^{11} \mathrm{~A}$ person who is bilaterally blind due to organic causes should still be able to do this simple test due to intact proprioception (Figure 4). Applying the same concept, the patient may be told to use their arm to point in a certain direction, and then asked to point in the same direction with the other arm; patients with NOVL will display difficulty pointing the subsequent arm in the same direction as the first. Also, ask the patient how many fingers are being held up; a patient with NOVL will report an incorrect number of fingers being displayed.

\section{Visual field - tunnel versus funnel vision}

Visual field abnormalities can provide additional evidence of nonorganic aetiology, in some cases. It can sometimes be difficult to accurately map out certain visual field defects using finger confrontation alone, for example, in bitemporal field defects and more formal testing may be required. ${ }^{29}$ One of the most common visual field deficits encountered in NOVL is a tunnel field, i.e., a constricted visual field restricting the usable field to central 5-10 degrees (Figure 5). Normal physiologic visual field is a 'funnel' that expands as you move further away from the target. This concept could be used in patients with unilateral or bilateral constricted fields. Visual field is first checked by confrontation at $1 \mathrm{~m}$ in all quadrants, to map out the approximate area of visual field. Then move away to $\sim 2-4 \mathrm{~m}$ (or the maximum distance that the examination room allows) and repeat. Under normal physiological fields (and even in patients with constricted fields from an organic cause), the area mapped should expand out, i.e., the patient should be able to detect your finger to a further periphery, as compared with $1 \mathrm{~m} .{ }^{5,11} \mathrm{~A}$ field that fails to expand is suggestive of NOVL. A tunnel visual field can also be demonstrated with a tangent screen. 
Figure 4: Test of proprioception

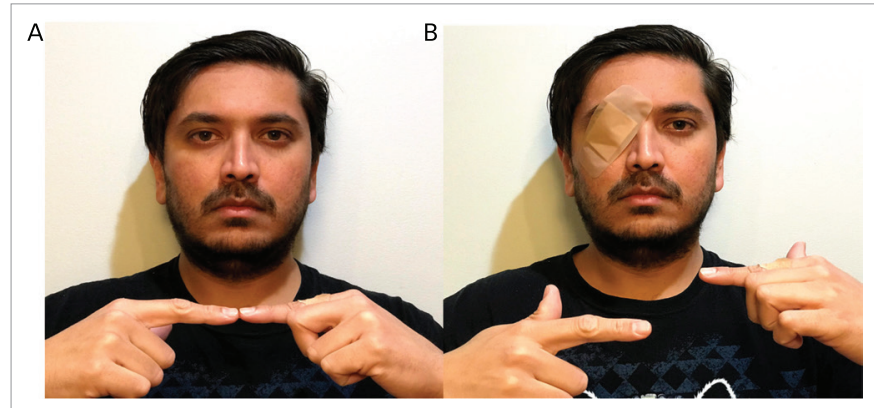

A. A person with unilateral/bilateral blindness should be able to touch tips of their fingers as shown in the image by virtue of intact proprioception; B. However, a patient with functional vision loss may show difficulty doing this after the good eye is patched stating inability to see.

Kerr et al. compared seven different methods of the confrontation test in a clinic setting, including using kinetic and static wiggling finger, and a kinetic and static red target. ${ }^{30}$ They concluded that the best method to detect visual field loss from neurologic causes was a $5 \mathrm{~mm}$ kinetic red target. The target should be moved from the peripheral non-seeing field across the midline while checking the patient's ability to see and interpret a change in colour/hue of the red target. ${ }^{30}$

Goldman kinetic visual-field tests, administered by a well-trained administrator, may be a more reliable technique for assessment. Patients with NOVL may show a constriction with non-physiologic overlap of isopters (Figure 5). They may claim to see the smaller, less-bright object at the same place as the larger, brighter test object. A continuous spiral or a jagged inconsistent star pattern may be produced.

On an automated perimeter, visual fields can be checked initially with a smaller target and then repeated with a larger target. However, automated perimetry is generally not helpful in the assessment of NOVL. Poor testing parameters, inconsistent responses and variable testing patterns do not differentiate NOVL from organic aetiology. A 'clover leaf', or rarely a square-like pattern, mapped out on automated perimetric testing is suggestive, but not diagnostic, of NOVL, and can be seen even in distracted test takers (Figure 6). ${ }^{17}$ In contrast a central scotoma on field testing is least likely to be from a NOVL, ${ }^{29,31}$ and should prompt the clinician to look further for underlying optic nerve or retinal pathology.

\section{Binocular visual field testing}

If one eye is truly non-seeing, and visual field is checked with both eyes open, the good eye will compensate for the missing field of the bad eye. In a patient with NOVL, on binocular field testing, the monocular field loss will persist on binocular testing. This test can be used even in a patient reporting constricted visual field or hemianopic field defect in one eye. On binocular field testing, if the proclaimed defect, i.e., constricted field or hemianopic defect, continues to persist, this is suggestive of NOVL, as the visual field with the seeing eye alone should produce a practically normal visual field under binocular conditions (Figures 7 and 8). The functional status of the good eye could be initially established with a monocular field of that eye prior to binocular testing. Monocular and binocular testing may be done with confrontation field testing, Goldman kinetic perimetry or automated perimetry.

\section{Ancillary testing}

\section{Visual evoked potential and electroretinogram}

Somers et al. report that pattern visual evoked potential (VEP) may be able to detect NOVL in children and help differentiate from organic visual loss. ${ }^{6}$ VEPS
Figure 5: Goldman kinetic perimetry demonstrating normal visual field and constricted visual fields in nonorganic vision loss
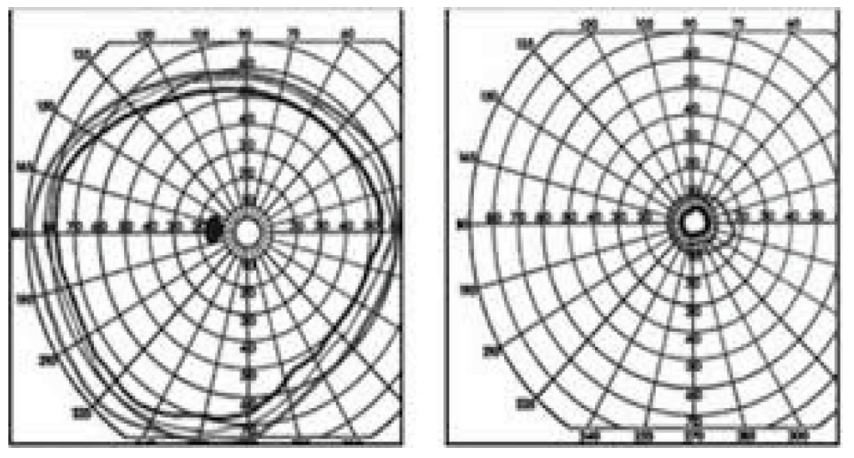

of normal and symmetric amplitude and latency in profound monocular visual loss can help the diagnosis of NOVL. This test can also be useful in a larger group of patients who have nonorganic overlay over underlying established vision loss, and who may exaggerate the extent of vision loss. ${ }^{32}$ VEP can give a gross correlation of the visual status. A VEP of $<5.77 \mu \mathrm{V}$ is closely consistent with legal blindness, i.e., <20/200 acuity, and could fairly help reduce suspicion for NOVL. ${ }^{32}$ Multifocal VEPS may demonstrate normal electrophysiological responses in the region of purported visual loss. However, an abnormal VEP is less helpful. It is important to note that VEP responses can be altered by the patient defocusing. This may be inapparent, even to a trained observer. On the other hand, normal VEP responses could be obtained in cortically blind patients.33,34

Pattern and multifocal ERG that produce a normal and symmetric response could argue against severe organic disease. Flash ERG predominantly measures the function the outer retinal layers, which may be abnormal in a patient with diffuse retinal dysfunction, but may be normal in a patient with more distal organic disease, such as optic neuropathy, chiasmal neuropathy, or retro chiasmal visual dysfunction, hence findings need to be cautiously interpreted. Neuroimaging, such as head and orbit CT/MRI, and vascular imaging, such as head and neck CT angiography/magnetic resonance angiography may help rule out compressive or vascular lesions; however, negative studies do not establish the diagnosis as functional. Optical coherence tomography (OCT) of the optic nerve and retina can identify anatomical pathology involving the optic nerve and retina; however, a normal OCT cannot rule out organic causes of vision loss or establish the diagnosis as nonorganic.

\section{Evaluation of index case}

The 17-year-old patient in the index case was asked to look at Snellen 20/100 line while a clear lens was introduced in front of his right eye (bad eye) and $a+12$ dioptre lens in front of the left eye (good eye), telling him that glasses were being tested to improve the left eye further. Unknowingly, he read down to the 20/30 line. A vertical four prism dioptre was introduced in front of his left eye after informing him that the prism will split his vision. He was asked to look at the 20/40 line and reported seeing two of each letter of equal clarity. The patient the wore red-green glasses and was presented with red and green letters of gradually increasing size on the Snellen's visual acuity chart. He was able to read the 20/20 line with red and green letters establishing good vision in the right eye.

This index case demonstrates a young male patient complaining of unilateral vision loss. However, when visual acuity was checked by fogging, the patient unknowingly read from his affected eye while 

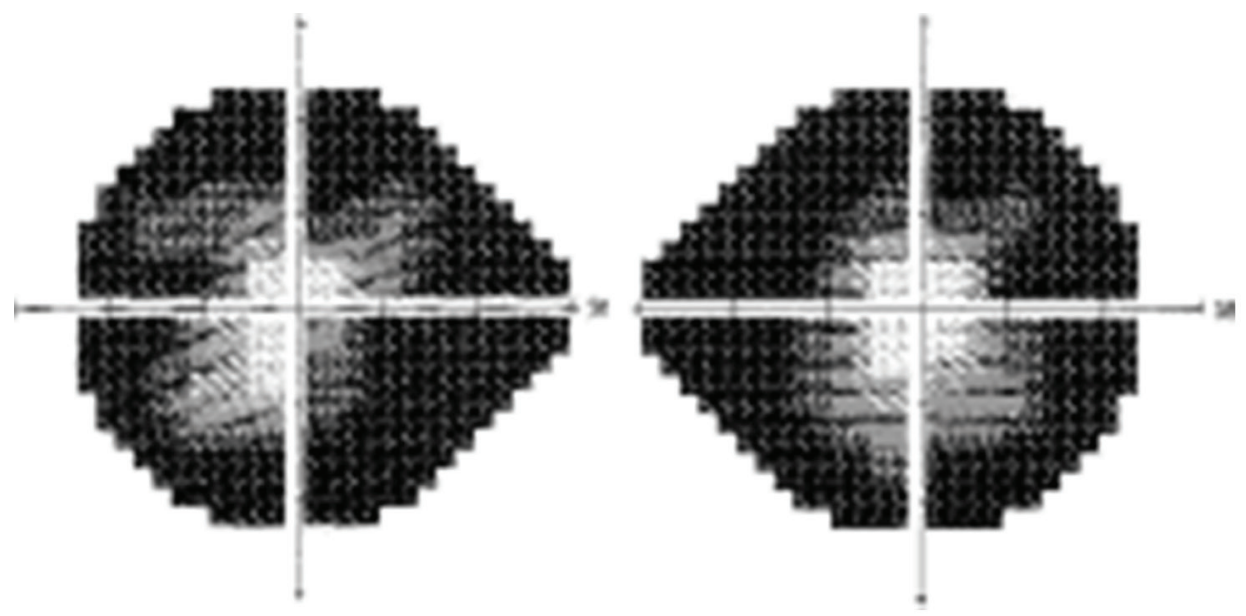

CENTRAL 24-2 THRESHOLO TEST

FIXATION MONITOR: GAZE/BLINOSPOT
FIXATION TRRCET: CENTRRL
FIXATION LOSSES: $8 / 17$
FALSE POS ERRDRS: $8 \%$
FALSE NEG ERRDRS: $33 \%$
TEST OURATION: $87: 33$
TEST OURATION: 07:33 FOVEA: 33 OB
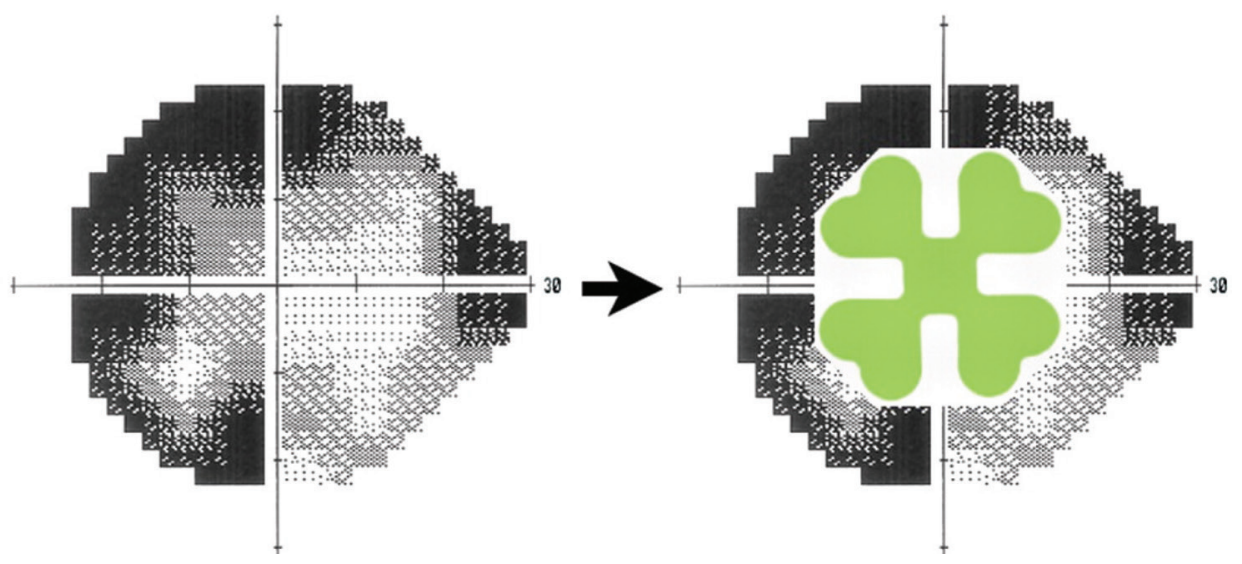

the good eye was fogged with $a+12$ dioptre lens. When a vertical prism was placed in front of his left eye and he was asked to read the Snellen's chart, he reported diplopia of letters of equal clarity, suggesting good vision in the right eye. If he had complete vision loss in one eye, he would have seen a single row of letters, and in case of poor vision in one eye the second image would have been blurred to the degree of vision loss. On colour vision testing, the first plate was a test plate, and is capable of being read/interpreted even by a person with colour-vision defect. With his 20/100 vision we would expect the patient to see the pattern on the test plate. Again, with red-green testing he unknowingly demonstrated good visual acuity in the right eye.

During visual field testing, in patients with organic causes of a constricted visual field, we would expect a physiologic 'funnel field' - one that would expand as we move away from patient. However, presence of a tunnel nonexpanding field is more suggestive of NOVL. On Humphrey's visual testing, the right eye of our patient showed a constricted clover-leaf field. In a person with unilateral organic vision loss, we expect the visual field from the other eye to compensate for loss from the poor eye, and expect the field with both eyes open to be normal. However, in our patient it remained constricted in the right eye. The rest of the ophthalmology examination and extensive testing in the past have been unrevealing. Repeating these tests, unless there is a strong suspicion for change, would not be fruitful. In this case, the first step was to initiate gentle discussion with the patient and his mother regarding the facts of the tests we had conducted. During follow-up, repeat vision checking is recommended to provide reassurance and encouragement that the condition is reversible.

\section{Pitfalls - where organic disease can be missed}

It is potentially easy to label a patient with NOVL; however, this can lead to devastating consequences if the patient actually has a treatable underlying cause that was missed. Some patients that appear to have a normal structural ophthalmic examination, may in fact have intracranial mass lesions, which can go undetected on CT scan and non-contrast MRI. A finger confrontation test could have a sensitivity as low as $20 \%$ in cases of bitemporal hemianopia, and hence an inability to detect a field defect by confrontation alone; therefore, it should not be used to rule out an organic cause of vision loss..$^{29}$ Homonymous hemianopia could be 
Figure 7: Monocular visual field testing with Goldman perimetry shows right temporal field loss that persists on binocular testing suggesting nonorganic visual loss

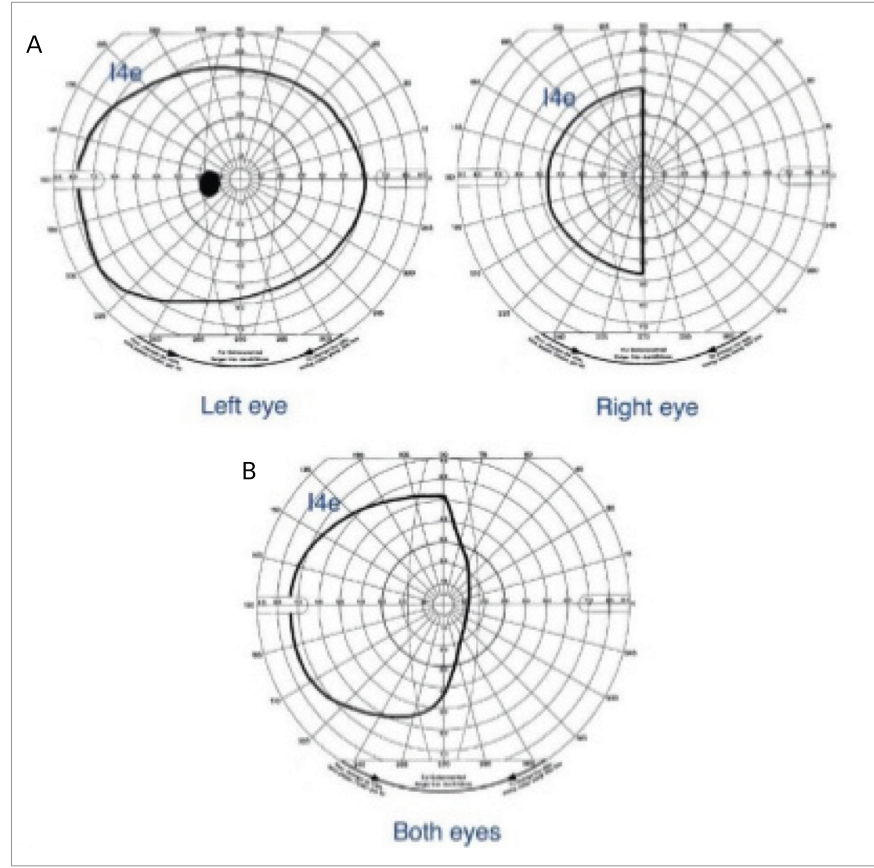

missed in up to $30 \%$ of patients..$^{30}$ Some patients with pituitary or frontal lesions may have accompanying psychiatric changes that could also add to the error in judgement. The clinician must also be cautious while evaluating patients with known psychiatric disease to not quickly dismiss complaints of visual loss as functional. Scott and Egan, in their paper, report a patient with persistent poor bilateral acuity who had normal visual field, colour vision, contrast sensitivity, stereopsis and structurally normal eye, but subsequently was diagnosed with cone dystrophy after multifocal ERG. ${ }^{35}$

The following are a few circumstances where organic vision loss may be mistaken as nonorganic:

- Retrobulbar optic neuritis where the fundus appears to be normal and RAPD may be subtle or missed

- Leber's hereditary optic neuropathy where there is acute or subacute loss of central vision in young males affecting both eyes simultaneously or sequentially. Interestingly, in unilateral presentation, RAPD could be absent and presence of family history of similar condition may be missed

- Traumatic optic neuropathy where unilateral or bilateral vision loss occurs post trauma, and varies from mild loss to total blindness. RAPD may be absent if there is bilateral symmetrical involvement. subtle RAPD may be missed in unilateral cases

- Cases of optic nerve glioma where there is slowly progressive vision loss, with subtle RAPD and proptosis that is not obvious

- Autosomal dominant optic atrophy, the most common hereditary optic neuropathy, where there is bilateral slowly progressive loss of vision and optic disc pallor is not initially apparent

- Optic tract lesions which are difficult to pick on clinical testing or even imaging

- Stargardt disease, the most common inherited single-gene retinal disease, where ophthalmology evaluation may overlook yellow flecks of lipofuscin deposits in the macula
Figure 8: Esterman's binocular automated perimetry demonstrating a right temporal defect in a subject reporting monocular right temporal field loss

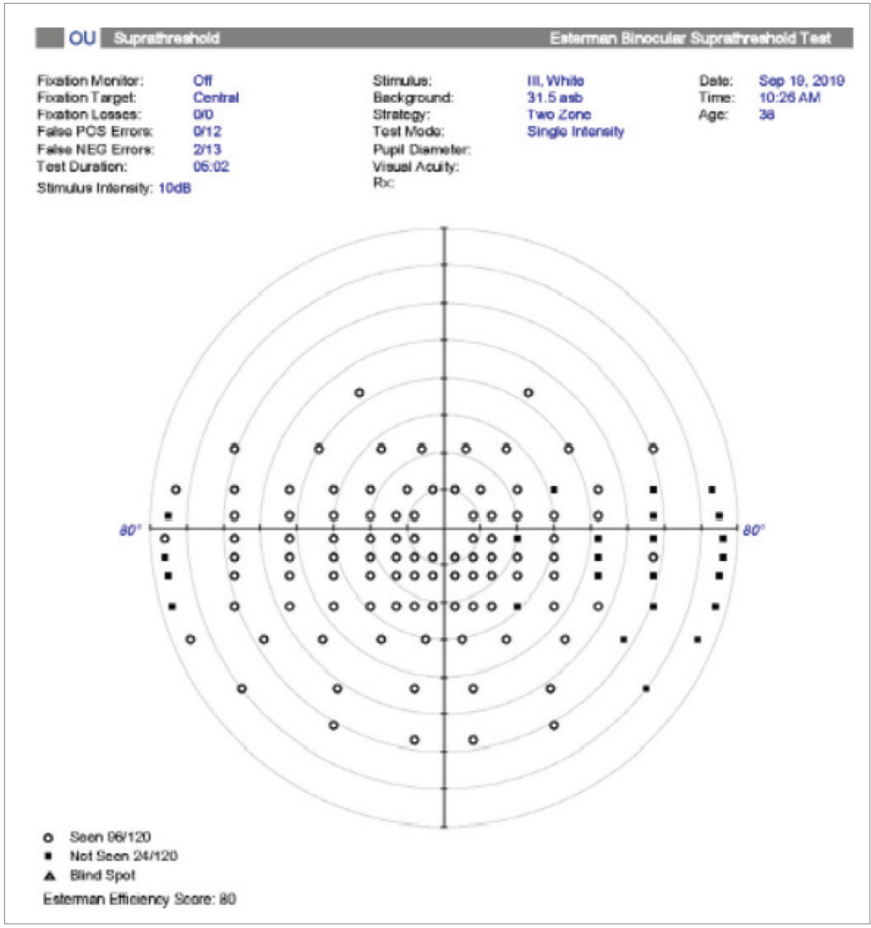

- Cone dystrophy where fundus examination looks normal in early stages

- Occult macular dystrophy that can result in central loss of vision without a RAPD, and can be easily missed on fundus examination. Occasionally further testing with OCT can reveal disruption in the anatomy of macula

- Amblyopia, the most common cause of monocular blindness where it is unusual to have RAPD

- Paraneoplastic syndromes affecting the optic nerve or retina, where patients can have a normal fundus examination and head CT/MRI

- Visual variant of Alzheimer's, a neurodegenerative syndrome where there is posterior cortical atrophy, and patients have a structurally normal eye examination

- In posterior hemisphere strokes involving the retrochiasmal pathway, where the eye looks structurally intact and homonymous hemianopia can be missed on confrontation visual fields

\section{How to deal with a nonorganic vision loss in the clinic}

The evaluation of NOVL can be time-consuming, tedious and can potentially frustrate providers. Patients may need several visits before a diagnosis can be established; such patients may need psychiatric evaluation, psychotherapy and counselling. However, patients with NOVL/FVL may not have as high an incidence of psychiatric disease as previously assumed. ${ }^{9-12}$

Yeo et al. mention that being open to the patient and their family, involving them in the care of the patient, and discussing with them the positive tests, such as a positive response on the optokinetic drum, may help encourage or motivate the patient and their family. ${ }^{36}$ An optokinetic stimulus can be obtained from YouTube or certain phone applications, and the family can be encouraged to use it at home regularly and acknowledge an improvement in the patient's visual status. Regular follow-up appointments may work as therapeutic sessions, during which, patients may be encouraged. This may also help build confidence in the 
patient rather than just labelling them as functional. Refusing followup appointments and telling the patient there is nothing wrong with their vision and therefore nothing that can be done for them, can only frustrate them further. This may make them go to a new physician for a repeat cycle of tests.

Stone and Edwards suggest that some of the strategies utilised in the diagnosis of functional deficit could also be applied to NOVL. ${ }^{37}$ Once it has been established that there are no defects or damage to the eyes, it is beneficial to diagnose/term the condition as NOVL, rather than tell the patient there is nothing wrong. The patient could be told that this is not unusual or abnormal, and that it may be explained by a processing error in the brain rather than structural damage to the eyes, i.e., their eyes can see, but their brain is unable to process the information. This could be used to reassure the patient that their problem is potentially reversible, and they need to re-establish the transmission error. ${ }^{37,38}$ Yeo et al. describe the use of transcranial magnetic stimulation and hypnotherapy to essentially increase awareness in the patient about visual perception. ${ }^{36}$

Mere suggestion, motivation and encouragement, together with repeated and frequent appointments may be necessary to build trust and confidence in the patient and suggest that there is a potential for improvement. ${ }^{8}$ This also reduces chances of 'doctor shopping' and multiple unnecessary tests. If the patient is a child, it is important to inform the parents of your findings. In older children, the diagnosis could also be discussed with them. Identification of underlying stressors in children may help with recovery and could be approached and discussed gently during clinic visits, either by individualised interviews with patient and family, or from questionnaires that could be filled out without the need for referral to psychiatry. ${ }^{8}$

\section{Prognosis}

There is limited literature on the recovery of vision loss and results are variable. Kathol et al., in a cohort study of 42 people with FVL (mean age 32 years), found that $45 \%$ regained normal visual function while $55 \%$ had persistent visual dysfunction at a mean follow-up of 53 months. ${ }^{910}$ Barris et al. found that $78 \%$ of 45 people in their cohort (mean age 25.9 years) showed improvement or normalisation of vision during a mean follow-up of 114 days, with the use of a timetable for recovery comprising reassurance and visual exercises. ${ }^{39}$ Sletteberg et al. found that $51 \%$ of the 41 people in their study reported good visual function, as opposed to $49 \%$ reporting poor visual function at a mean follow-up of 2 years. ${ }^{40}$ The latter two studies also found that patients aged $<16$ years were more likely to recover normal visual function compared with older people. Somers et al. reported that though there could be resolution of the visual symptoms, underlying psychiatric issues and psychosocial problems could emerge and may need gentle management. ${ }^{6}$

\section{Conclusion}

The role of the ophthalmologist and neuro-ophthalmologist is to establish NOVL/FVL and to prove normal visual function, while not missing organic visual loss. However, this can be difficult. One should always attempt to understand the potential underlying risk factors, if any, or underlying motivation. One should never confront the patient. Once a diagnosis is made, unnecessary tests or procedures should be avoided. $\square$
1. Chen CS, Lee AW, Karagiannis A, et al. Practical clinical approaches to functional visual loss. I Clin NeurosCl. 2007:14:1-7.

2. Toldo I, Pinello L, Suppiej A, et al. Nonorganic (psychogenic) visual loss inchildren: a retrospective series. I Neuroophthalmol. 2010;30:26-30.

3. Munoz-Hernandez AM, Santos-Bueso E, Saenz-Frances F, et al. Nonorganic visual loss and associated psychopathology in children. Eur J Ophthalmol. 2012:22:269-73.

4. Bose S, Kupersmith MJ. Neuro-ophthalmologic presentations of functional visual disorders. Neurol Clin. 1995; 13:321-39.

5. Moore Q, Al-Zubidi N, Yalamanchili S, Lee AG. Nonorganic visual Ioss in children. Int Ophthalmol Clin. 2012;52:107-23.

6. Somers A, Casteels K, Van Roie E, et al. Non-organic visual loss in children: prospective and retrospective analysis of associated psychosocial problems and stress factors. Acta Ophthalmol. 2016;94:e312-6.

7. Lim SA, Siatkowski RM, Farris BK. Functional visual loss in adults and children patient characteristics, management, and outcomes. Ophthalmology. 2005:112:1821-8.

8. Catalano RA, Simon JW, Krohel GB, Rosenber PN. Functional visual loss in children. Ophthalmology. 1986;93:385-90.

9. Kathol RG, Cox TA, Corbett JJ, et al. Functional visual loss: II. Psychiatric aspects in 42 patients followed for 4 years. Psychol Med 1983:13:315-24.

10. Kathol RG, Cox TA, corbett JJ, Thompson HS. Functional visual loss. Follow-up of 42 cases. Arch Ophthalmol. 1983;101:729-35. 1. Bruce BB, Newman NJ. Functional visual loss. Neurol Clin. 2010;28:789-802.

12. Kathol RG, Cox TA, Corbett JJ, et al. Functional visual loss: I. A true psychiatric disorder? Psychol Med. 1983;13:307-14.

13. Bengtzen R, Woodward M, Lynn MJ, et al. The "sunglasses sign" predicts nonorganic visual loss in neuro-ophthalmologic practice. Neurology. 2008;70:218-21.

14. Levy NS, Glick EB. Stereoscopic perception and Snellen visual acuity. Am J Ophthalmol 1974:78:722-4.
15. Sitko KR, Peragallo $\mathrm{JH}$, Bidot $\mathrm{S}$, et al. Pitfalls in the use of stereoacuity in the diagnosis of nonorganic visual loss Ophthalmology. 2016;123:198-202.

16. Zinkernagel SM, Mojon DS. Distance doubling visual acuity test: a reliable test for nonorganic visual loss. Graefes Arch Clin Exp Ophthalmol. 2009:247:855-8.

17. Miller NR. Neuro-ophthalmologic manifestations of psychogenic disease. Semin Neurol. 2006:26:310-20.

18. Slavin ML. The prism dissociation test in detecting unilateral functional visual loss. J Clin Neuroophthalmol 1990:10:127-30.

19. Golnik KC, Lee AG, Eggenberger ER. The monocular vertical prism dissociation test. Am J Ophthalmol. 2004;137:135-7.

20. Finkelstein JI, Johnson LN. Relative scotoma and statokinetic dissociation (Riddoch's phenomenon) from occipital lobe dysfunction. Trans Pa Acad Ophthalmol Otolaryngol. 1989;41:789-92.

21. Vidal Y, Hoffmann M. Improvement of astatikopsia (Riddoch's phenomenon) after correction of vertebral stenoses with angioplasty. Neurol Int. 2012;4:e1.

22. Foroozan R, Lee AG. Don't get off the track. Surv Ophthalmol. 2018;63:437-44.

23. Al-Zubidi N, Ansari W, Fung SH, Lee AG. Diffusion tensor imaging in traumatic optic tract syndrome. J Neuroophthalmol. 2014;34:95-8.

24. Kowal KM, Rivas Rodriguez FF, Srinivasan A, Trobe JD. Spectrum of magnetic resonance imaging features in unilateral optic tract dysfunction. J Neuroophthalmol. 2017:37:17-23.

25. Moster ML, Galetta SL, Schatz NJ. Physiologic functional imaging in "functional" visual loss. Surv Ophthalmo 1996;40:395-9.

26. Silverman IE, Galetta SL, Gray LG. SPECT in patients with cortica visual loss. J Nucl Med. 1993;34:1447-51.

27. Wakakura M, Yokoe J. Evidence for preserved direct pupillary light response in Leber's hereditary optic neuropathy. $\mathrm{Br}$ Ophthalmol. 1995;79:442-6.
28. Levi L, Feldman RM. Use of the potential acuity meter in suspected functional visual loss. Am I Ophthalmo 1992;114:502-3.

29. Johnson LN, Baloh FG. The accuracy of confrontation visual field test in comparison with automated perimetry. J Natl Med Assoc. 1991;83:895-8.

30. Kerr NM, Chew SS, Eady EK, et al. Diagnostic accuracy of confrontation visual field tests. Neurology. 2010;74: $1184-90$.

31. Thompson JC, Kosmorsky GS, Ellis BD. Field of dreamers and dreamed-up fields: functional and fake perimetry. Ophthalmology. 1996;103:117-25.

32. Jeon J, Oh S, Kyung S. Assessment of visual disability using visual evoked potentials. BMC Ophthalmol. 2012;12:36

33. Howard JE, Dorfman L. Evoked potentials in hysteria and malingering. J Clin Neurophysiol. 1986;3:39-49.

34. Spehlmann R, Gross RA, Ho SU, et al. Visual evoked potentials and postmortem findings in a case of cortical blindness. Ann Neurol. 1977;2:531-4.

35. Scott JA, Egan RA. Prevalence of organic neuro-ophthalmologic disease in patients with functional visual loss. Am J Ophthalmol. 2003:135:670-5.

36. Yeo JM, Carson A, Stone J. Seeing again: treatment of functiona visual loss. Pract Neurol. 2019:19:168-72.

37. Stone 1 Edwards $M$. Trick or treat? Showing patients with functional (psychogenic) motor symptoms their physical signs. Neurology. 2012;79:282-4.

38. Stone J, Hoeritzauer I, Brown K, Carson A. Therapeutic sedation for functional (psychogenic) neurological symptoms. J Psychosom Res. 2014;76:165-8.

39. Barris MC, Kaufman DI, Barberio D. Visual impairment in hysteria. Doc Ophthalmol. 1992;82:369-82.

40. Sletteberg $\mathrm{O}$, Bertelsen $\mathrm{T}$, Høvding $\mathrm{G}$. The prognosis of patients with hysterical visual impairment. Acta Ophthalmol. 1989;67:159-63. 